\title{
Potential Linagliptin-Induced Renal Impairment
}

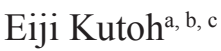

\begin{abstract}
It is known that linagliptin, a novel DPP-4 inhibitor, can be used in patients even with severe renal impairment. So far, there have been no reports describing the clinical presentations and features of a patient with linagliptin-induced renal toxicity. A 66-year-old Japanese man had developed nausea and elevations of creatinine and potassium levels at 4 weeks after switching from sitagliptin to linagliptin. No emesis or diarrhea was noted. These renal parameters had slowly recovered after switching-back to sitagliptin. This slow recovery process may be due to the very long half-life of this drug. Although no single case report can prove case or effect of drugs, it is still safe that physicians carefully monitor renal function upon administration of linagliptin, especially in those with certain degrees of chronic kidney disease.
\end{abstract}

Keywords: DPP-4 inhibitor; Linagliptin; Sitagliptin; Renal impairment

\section{Introduction}

Current therapeutic agents for type 2 diabetes (T2DM) may be limited by adverse events, drug-drug interactions, or contraindication/requirement for dose adjustment in patients with renal or hepatic impairment. Especially since the subjects with T2DM frequently have progressing renal deterioration, there is a particular need for drugs that can be safely and effectively used when renal function is declining.

\footnotetext{
Manuscript accepted for publication July 10, 2012

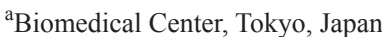

${ }^{\mathrm{b}}$ Department of Internal Medicine, Higashi Totsuka Memorial Hospital, Yokohama, Kanagawa, Japan

${ }^{\mathrm{c}}$ Corresponding address: Eiji Kutoh, Biomedical Center, 1-5-8-613

Komatsugawa, Edogawa, Tokyo, 132-0034, Japan.

Email: ekuto@excite.com

doi: http://dx.doi.org/10.4021/jmc807w
}

Dipeptidyl peptidase 4 (DPP-4) inhibitors are promising new therapeutic options for the treatment of T2DM. They block degradation of incretin by DPP-4, thereby possessing a wide range of physiological effects associated with improved glycemic control including stimulation of glucose-dependent insulin secretion and suppression of glucagons secretion [1]. However, the most widely used DPP-4 inhibitor, sitaglitin, is contra-indicated in patients with severe renal impairment [2], while alogliptin or vildagliptin needs dose adjustment or careful attention with such patients in Japan [3,4]. One of the newest DPP-4 inhibitors on the market, linagliptin, has distinct pharmacokinetic properties. Its elimination is through non-renal routes $[5,6]$. Thus, it can be used regardless of the degree of renal impairment without dose adjustment and/or monitoring renal function $[5,6]$. Thus, linagliptin may offer an appropriate option for those in whom metformin and/or other DPP-4 inhibitors are either contraindicated or require dose adjustment because of moderate to severe renal impairment. However, this report presents a case in which linagliptin was most probably responsible for the development of renal impairment with a 66-year-old Japanese patient.

\section{Case Report}

A 66-year-old Japanese man has been visiting the outpatient division of Department of Internal Medicine at Higashi Totsuka Memorial Hospital, Yokohama, Japan for the treatment of hypertension, chronic kidney disease (CKD), hyperuricemia and T2DM. He was taking dilazep $100 \mathrm{mg}$ three times daily, kremezin (AST-120) $2 \mathrm{~g}$ three times daily, allopurinol $100 \mathrm{mg}$ once daily and imidapril $5 \mathrm{mg}$ once daily in the last 2 years. Apart from these, he was using combinatory inhalation of salmeterol and fluticasone in the case of asthma events. T2DM was diagnosed many years ago and was initially treated with diet and exercise alone. However, since his glycemic control began deteriorating, he commenced DPP-4 inhibitor therapy with sitagliptin $25 \mathrm{mg}$ once daily in June 2011(month 0, Fig. 1). At that time, his values in HbA1c, body weight, body height and blood pressure (systolic/diastolic) were $8.0 \%, 70.4 \mathrm{~kg}, 165 \mathrm{~cm}$, and $126 / 76 \mathrm{mmHg}$, respectively. His liver function was normal and he had no dia- 


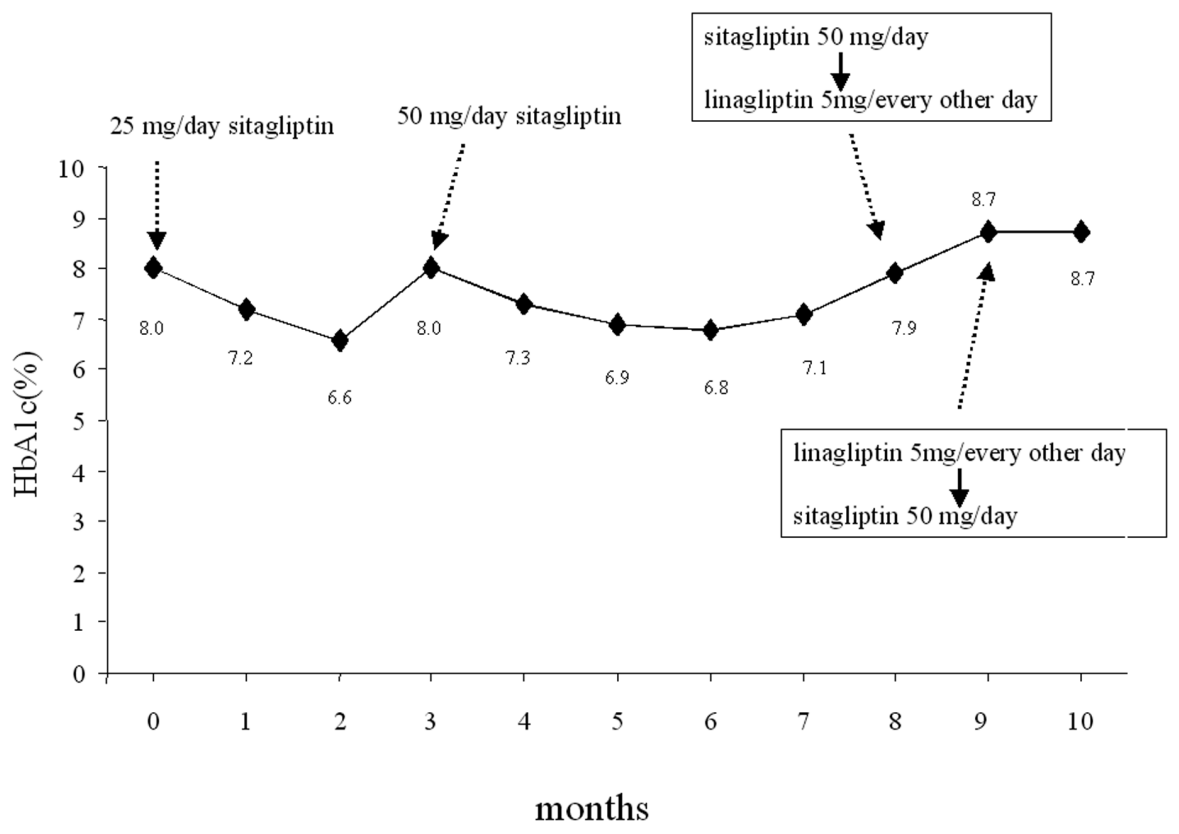

Figure 1. Change of glycemic parameters. Sitagliptin $25 \mathrm{mg} /$ day was initiated in June, 2011 (month 0). The following therapeutic modifications and changes of $\mathrm{HbA} 1 \mathrm{c}$ levels are shown.

betic retionopathy or diabetic polyneuropathy. The HbA1c values were assessed by the JDS standardization [7] throughout this manuscript. To convert these into the National Glycoprotein Standardization Program (NGSP), add $0.4 \%$ to the indicated values [7].

Sitagliptin $25 \mathrm{mg}$ once daily was initially effective in the first 3-4 months but after this period, the HbAlc levels had a tendency to increase, therefore, the dose of this drug was increased to $50 \mathrm{mg}$ once daily in September 2011 (month 3, Fig. 1). The HbA1c levels decreased in the next several months, however, after a while they started to increase again (Fig. 1). This secondary failure of sitagliptin is occasionally observed (E. Kutoh, personal communications). In contrast to this, the CKD control (assessed by creatinine: CRE and potassium: K) was relatively under fair range and no significant variations of these parameters were noted (Fig. 2). Also, no changes in the levels of blood pressure or body weight were observed (data not shown). In February 2011 (month 8, Fig. 1), sitagliptin $50 \mathrm{mg}$ once daily was switched to linagliptin $5 \mathrm{mg}$ every other day. The other drugs were unchanged. At 4 weeks after the initiation of linalgliptin, the patient complained of slight nausea but no emesis or diarrhea. Blood analysis had revealed that the CRE and $\mathrm{K} \mathrm{lev-}$ els significantly increased (Fig. 2) and the glycemic control had worsened (Fig. 1). The levels of liver enzymes were unchanged (results not shown).

Immediately, discontinuation of linagliptin and switching-back to sitagliptin $50 \mathrm{mg}$ once daily were undertaken. The other drugs were unchanged. At 4 weeks after the discontinuation of linagliptin, disappearance of nausea and reductions of these renal parameters (CRE and $\mathrm{K}$ ) were observed (Fig. 2). These renal parameter levels further decreased (although very slowly) after this period, but not to the previous range before the start of linagliptin even at 8 weeks after the discontinuation of linagliptin (Fig. 2).

\section{Discussion}

This case report emphasizes the possibility of acute renal impairment induced by linagliptin. The impaired renal function (assessed by significant elevations of CRE and K levels) and nausea at 4 weeks after the initiation of linagliptin may be typical of an idiosyncratic drug reaction. However the specific pathophysiology responsible for this potential adverse event remains unknown. As described in Introduction, linagliptin is excreted through non-renal routes; thereby it can be used in patients even with severe renal impairment. However, a possibility that this drug can affect kidney through an indirect way cannot be excluded.

With the cessation of linagliptin and switching back to sitagliptin, the patient's condition improved and the CRE and $\mathrm{K}$ levels had slowly declined (Fig. 2). The renal impairment in this case appears to be reversible. However, if the reduction in renal blood flow is severe or prolonged, ischemic damage to the tubles may occur, resulting in serious consequences (e.g. acute tubular necrosis). The pathophysiology of the above mentioned clinical manifestations may be described as follows: in mild to moderate impaired renal conditions, glomerular filtration rate (GFR) and renal perfu- 


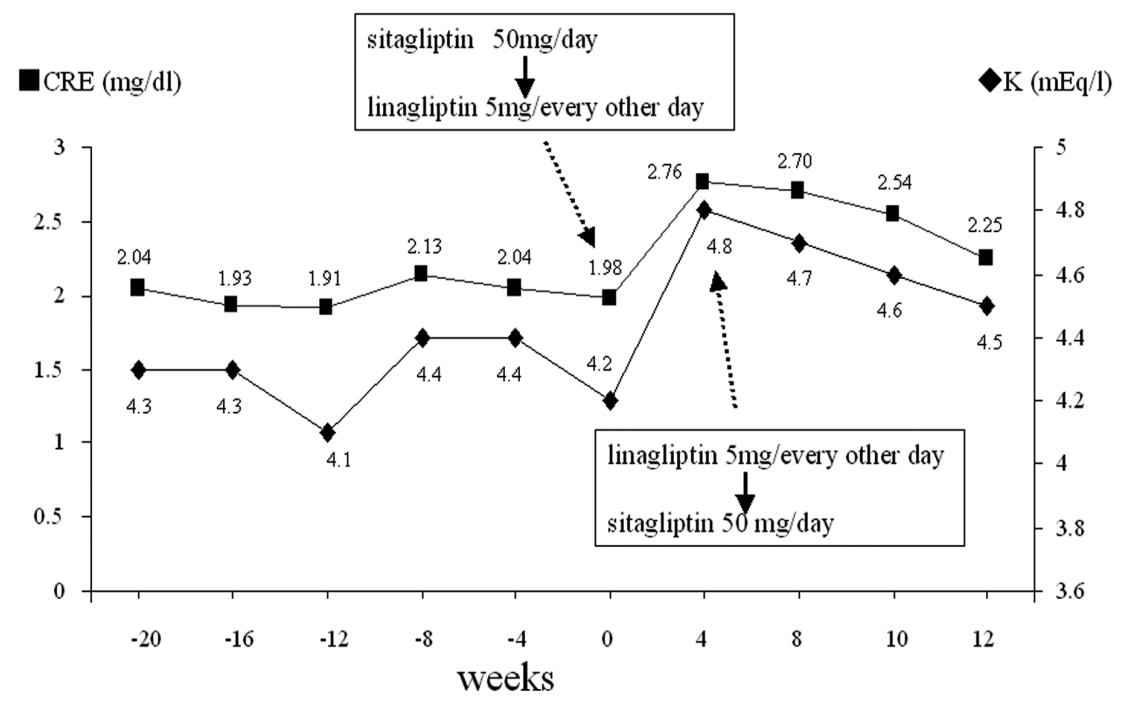

Figure 2. Change of renal parameters. Sitagliptin $50 \mathrm{mg} /$ day was switched to linagliptin $5 \mathrm{mg} / \mathrm{every}$ other day in February 2012 (week 0) and linagliptin $5 \mathrm{mg} /$ every other day was switched back to sitagliptin $50 \mathrm{mg} /$ day at week 4. The changes of CRE and $\mathrm{K}$ levels are shown before and after these therapeutic switches.

sion are maintained by the autoregulatory mechanisms and as a consequence, the renal function remains normal. However, if the renal blood flow is severely reduced, the autoregulatory mechanisms that enhance the hydrostatic pressure at the glomerulus in response to reduced GFR and/or renal perfusion are insufficient to overcome this situation. Finally acute renal failure might occur. In this regard, markers for tubular injury (e.g. beta2-microglobulin or N-acetyl-beta-Dglucosaminidase) should have measured.

The possibility that other drugs (e.g. imidapril) used by this patient caused this adverse event cannot be fully ruled out. Indeed, some patients using renin angiotensin aldosterone (RAAS)-inhibitors (ACE-inhibitors and ARB) may develop impaired renal function $[8,9]$. The underlying mechanism of this phenomenon is that RAAS inhibitors block the normal pathways to protect renal perfusion in the event of hypovolemia. However, since the patient was taking imidapril for several years prior to sitagliptin or linagliptin and the CRE or $\mathrm{K}$ levels were stable without any significant variations (Fig. 2), it is unlikely that this drug (imidapril) had caused the renal impairment in question. However, it can be possible that the multiple drug-drug interactions had caused this observed renal impairment.

One of the most common causes of sudden elevation of renal parameters (e.g. CRE or K) apart from impaired renal function is dehydration. Most probably, dehydration was not the cause in this case. The episodes of nausea were significant, but emesis and diarrhea were not observed. In the absence of an obvious diarrhea, emesis or loss of fluid and considering the temporal sequence of events, this case suggests a causal link between the initiation of linagliptin and the development of impaired renal function. Further, if the impaired renal function was due to dehydration, the recovery process of the renal parameters (CRE or $\mathrm{K}$ ) should be faster than the period observed in this case report (Fig. 2).

Although different DPP-4 inhibitors share a common mechanism of action and similar glycemic efficacies, they greatly differ in their chemical features [10]. Linagliptin, for example, has a xantine-based structure, which may be responsible for its long terminal elimination half-life, more than 100 hours [11]. The long half-life of linagliptin may be useful in some occasions, for example, for patients who occasionally miss their medication, compared with the shorter half-life of siagliptin or saxagliptin, 12.4 and 2.5 hours, respectively [11]. However, at the same time, this long-half life may be a disadvantage. If any unfavorable adverse events happen, this drug may retain in the body for a very long period. The long recovering period of renal function after discontinuation of linagliptin in this report may reflect this feature. Under serious conditions, hemodialysis may be the only solution to remove this drug from the body.

Although it is said that linagliptin can be used with any degrees of renal impairment without dose adjustment and/or monitoring renal function $[5,6]$, the result of this case report might indicate that it is still safe to monitor the renal parameters in patients with certain degrees of CKD.

\section{Conflict of Interest}

None.

\section{Declaration of Funding}

None. 


\section{References}

1. Lovshin JA, Drucker DJ. Incretin-based therapies for type 2 diabetes mellitus. Nat Rev Endocrinol. 2009;5(5):262-269.

2. Sitagliptin drug information www.info.pmda.go. jp/ downfiles/ph/GUI/170050_39690 10F1034_2_11G.pdf.

3. Alogliptin drug information http://www.takedamed. com/hpdr/ rootDir/ medicine /medicine Detail.jsp? MEDICINE CODE=140 http: //www. takedamed. com/ hpdr/rootDir/medicine/nesina/index.jsp.

4. Vildagliptin drug information http://www. info.pmda.go.jp /downfiles/ph/ GUI/ 300242_3969011F1020_1_08G.pdf.

5. Forst T, Pfutzner A. Linagliptin, a dipeptidyl peptidase-4 inhibitor with a unique pharmacological profile, and efficacy in a broad range of patients with type 2 diabetes. Expert Opin Pharmacother. 2012;13(1):101-110.

6. Neumiller JJ. Pharmacology, efficacy, and safety of linagliptin for the treatment of type 2 diabetes mellitus. Ann
Pharmacother. 2012;46(3):358-367.

7. Tominaga M, Makino H, Yoshino G, Kuwa K, Takei I, Aono Y, Hoshino T, et al. Japanese standard reference material JDS Lot 2 for haemoglobin A1c. II: Present state of standardization of haemoglobin A1c in Japan using the new reference material in routine clinical assays. Ann Clin Biochem. 2005;42(Pt 1):47-50.

8. Scherpbier ND, de Grauw WJ, Wetzels JF, Vervoort GM. [Acute renal failure due to RAAS-inhibitors combined with dehydration]. Ned Tijdschr Geneeskd. 2010;154:A1548.

9. Giovanna L, Francesca V, Roberto P. RAAS inhibition and renal protection. Curr Pharm Des. 2012;18(7):971980.

10. Baetta R, Corsini A. Pharmacology of dipeptidyl peptidase-4 inhibitors: similarities and differences. Drugs. 2011;71(11):1441-1467.

11. Freeman MK. Efficacy and safety of linagliptin (tradjenta) in adults with type- 2 diabetes mellitus. $\mathrm{P}$ T. 2011;36(12):807-842. 